Relations industrielles

Industrial Relations

\title{
Méthodes d'appréciation des cadres, par H.J. Kreitz, Paris, Les Éditions de l'organisation, 1971, 135 pp.
}

\section{Laurent Bélanger}

Volume 29, numéro 2, 1974

URI : https://id.erudit.org/iderudit/028519ar

DOI : https://doi.org/10.7202/028519ar

Aller au sommaire du numéro

Éditeur(s)

Département des relations industrielles de l'Université Laval

ISSN

0034-379X (imprimé)

1703-8138 (numérique)

Découvrir la revue

Citer ce compte rendu

Bélanger, L. (1974). Compte rendu de [Méthodes d'appréciation des cadres, par H.J. Kreitz, Paris, Les Éditions de l'organisation, 1971, 135 pp.] Relations industrielles / Industrial Relations, 29(2), 414-415.

https://doi.org/10.7202/028519ar

Tous droits réservés @ C Département des relations industrielles de l'Université Laval, 1974
Ce document est protégé par la loi sur le droit d'auteur. L’utilisation des services d'Érudit (y compris la reproduction) est assujettie à sa politique d'utilisation que vous pouvez consulter en ligne.

https://apropos.erudit.org/fr/usagers/politique-dutilisation/ 
services de personnel. Les auteurs devraient reviser les critères qui président au partage des responsabilités et de l'autorité au sein des organisations de travail et nous fournir une représentation symbolique d'un service de personnel qui est à jour au plan de la structure et $\mathrm{du}$ fonctionnement.

$\mathrm{Au}$ dernier chapitre, les auteurs proposent une nouvelle approche, celle de la gestion par communication. Je ne vois rien de tellement nouveau dans cette approche puisque depuis vingt ans, c'est-à-dire depuis la diffusion des résultats de recherche dans le domaine des relations humaines et des styles d'administration, on ne cesse de rappeler l'importance de la communication au sein des organisations et de la diffusion de l'information à tous les niveaux, l'information nécessaire à l'exécution des tâches et l'information qui permettrait aux individus et aux groupes de se situer dans la grande entreprise.

Malgré ces quelques faiblesses, l'ouvrage demeure valable au plan de la présentation des techniques et de quelques principes fondamentaux. La liste de questions qui suit chaque chapitre permettra aux praticiens de procéder à une évaluation de leur travail, de même que les tests de motivation, de style de supervision leur permettront de mieux se connaitre. Ces tests sont suivis d'une série de cas pratiques qui peuvent être utiles aux étudiants qui suivent un premier cours dans ce domaine. La plupart des cas sont présentés sous forme de jeux de rôle qui s'adaptent bien à une classe de 20 personnes ou moins. De plus, chaque chapitre est suivi d'une bibliographie qui fait état des meilleurs ouvrages sur chacun des thèmes retenus par les auteurs.

Puisque ce volume se veut avant tout une synthèse des pratiques existantes et qu'il cherche à couvrir tous les aspects de la gestion du personnel et des relations patronales-ouvrières, le lecteur ne doit pas s'attendre à y retrouver des notions approfondies sur des sujets aussi importants que la grève, la législation $\mathrm{du}$ travail, les structures et le fonctionnement du mouvement syndical au Québec. C'est par la lecture de d'autres ouvrages qu'il arrivera à une connaissance plus approfondie de ces sujets.

Université Laval
Méthodes d'appréciation des cadres, par H.J. Kreitz, Paris, Les Editions de l'organisation, 1971, $135 \mathrm{pp}$.

C'est un recueil pratique qui s'éloigne peu des sentiers battus en matière d'évaluation des cadres. Après avoir décrit la nature du processus d'évaluation, ses avantages et ses difficultés, l'auteur procède à l'établissement d'une liste de crtères qui peuvent être utilisés pour juger de la valeur d'un cadre et, en particulier, de son rendement. Un deuxième chapitre traite des critères qu'on utilise habituellement au cours de l'évaluation. Chaque critère est accompagné d'une échelle de points qui permet de noter le personnel de cadre au plan du rendement, des connaissances, des habiletés professionnelles, etc. Chaque poste de travail doit, au préalable, être évalué en fonction des mêmes critères de façon à préciser les exigences théoriques en termes de points. Au cours de l'évaluation, les qualifications actuelles de chaque cadre sont notées et comparées aux qualités requises pour le poste. En plus de déboucher sur une notation de l'individu, l'évaluation permet de saisir le décalage entre les qualifications acquises et les exigences théoriques pour chaque poste. Les critères ainsi que les échelles qui les accompagnent peuvent être intégrés dans un même formulaire qui servirait d'instrument unifome au sein d'une même organisation de travail. Le troisième chapitre traite de l'utilisation de la technique d'évaluation par les évaluateurs ou les descripteurs, un terme utilisé par l'auteur dont le choix n'est pas heureux puisque le concept de discripteur sert à qualifier un critère et non la personne qui fait l'évaluation. Le dernier chapitre traite également d'une méthode d'évaluation des fonctions de direction en termes de niveaux de responsabilités et de formation scolaire. Ce chapitre m'apparaît plutôt comme un hors d'oeuvre ou une annexe puisqu'il m'est difficile de saisir la manière dont il s'intègre au reste du volume et l'usage dont on peut en faire en matière d'évaluation.

Puisque les cadres, comme tous les employés d'ailleurs, désirent savoir où ils en sont tant dans leur travail que dans leur carrière, l'évaluation. périodique devient une activité importante. $\mathrm{Ce}$ petit volume peut servir de guide, plus ou moins fiable, cependarit, puis- 
qu'il comporte quelques faiblesses majeures. Les critères retenus sont apparemment tous d'égale importance; ils ne comportent aucune pondération. De plus, l'auteur ne distingue pas entre l'appréciation du potentiel d'un candidat et l'appréciation de son rendement. A mon avis, ces deux processus sont distincts et font l'objet de deux évaluations distinctes. Le premier a pour objectif de déceler les forces et les faiblesses d'un cadre en vue de lui tracer un programme de développement personnel au cours de sa carrière, alors que le second a pour objectif de juger du rendement au travail pour apporter les correctifs qui s'imposent en courte période et pour attribuer des augmentations au mérite s'il y a lieu. Dans l'ensemble, le volume n'apporte aucune contribution nouvelle aux connaissances qu'on retrouve déjà dans les bons manuels de direction du personnel.

Université Laval

Laurent BELANGER

Federalism and Policy Development : The Case of Adult Occupational Training in Ontario, by J. Stefan Dupré, David M. Cameron, Greame $\mathrm{H}$. McKechnie and Theodore B. Rotenberg, Toronto, University of Toronto Press, 1973, 248 pp.

Alors qu'on se plaît souvent à l'étranger et ce surtout aux Etats-Unis, à vanter les mérites du système canadien de formation professionnelle, il n'existe malheureusement que très peu d'écrits canadiens sur ce sujet et sur la plupart des autres programmes de main-d'oeuvre à part le programme québécois de reclassement, Federalism and Policy Development vient sûrement combler un vide non excusable.

C'est en 1966, comme l'indiquent les auteurs dans leur préface, que le gouvernement canadien a abruptement mis fin à une vieille relation de prêts-conditionnels aux provinces dans le domaine de la formation technique et professionnelle. On en vint alors à un nouvel arrangement qui consistait pour Ottawa à acheter des provinces la formation professionnelle des adultes (F.P.A.) et ce comme partie intégrale d'une politique de main-d'oeuvre. Fondamentalement, ce livre examine les circonstances entourant cette transition et en examine les suites plus particulièrement pour l'Ontario. Cependant, il est important de retenir au départ que cet ouvrage n'a pas comme but premier de présenter et de réfléchir sur la formation professionnelle. Celle-ci n'est en fait qu'instrumentale pour permettre l'atteinte du but réel que les auteurs poursuivent i.e. examiner le développement de politiques et de programmes dans un cadre fédéraliste. Cette distinction que nous venons de faire est importante à notre avis car tout au long de cet ouvrage le lecteur se surprend maintes fois à confondre ces deux objectifs et même à voir dans cet ouvrage un traité sur la formation professionnelle au Canada, ce que fondamentalement il n'est pas. Un autre problème suscité par cette confusion d'objectifs serait le suivant: on a souvent l'impression que les auteurs confondent formation professionnelle et politique de main-d'oeuvre. Même si en fait cela n'est sûrement pas le cas, il n'en demeure pas moins qu'il aurait été très utile que les auteurs situent le programme de formation professionnelle dans la politique canadienne de main-d'oeuvre. Après tout, les efforts du gouvernement fédéral en main-d'oeuvre ne se situent pas seulement en formation professionnelle. Finalement, notons que nulle part dans le texte les auteurs expliquent leur conception d'une politique de main-d'oeuvre. Même si instrumentale à leur étude, cette présentation aurait sûrement été utile. Fondamentalement alors, cet ouvrage est beaucoup plus un examen de certaines relations de pouvoirs exercées entre divers niveaux de gouvernement (Fédéral et Ontario) à l'occasion du développement de politiques et de programmes. La formation professionnelle y joue donc un rôle secondaire. Ce n'est en fait qu'un exemple et la structure même de cet ouvrage appuie cet énoncé.

Ce livre comporte trois parties principales : transition, opération et conclusions, le raisonnement étant d'expliquer de quoi on est parti, à quoi on en est arrivé et vers quoi on se dirige dans le domaine de la formation professionnelle au Canada et de voir dans ce passage la nature et les conséquences de la double présence gouvernementale. C'est pourquoi la première partie de cet ouvrage, la transition, s'arrête sur les relations fédérales-provinciales, sur les efforts déployés pour esquisser une poli- 